\title{
Averaging anisotropic cosmologies
}

\author{
John D. Barrow ${ }^{1 *}$ and Christos G. Tsagas ${ }^{2 \dagger}$ \\ ${ }^{1}$ DAMTP, Centre for Mathematical Sciences, University of Cambridge \\ Wilberforce Road, Cambridge CB3 0WA, UK \\ ${ }^{2}$ Section of Astrophysics, Astronomy and Mechanics, Department of Physics \\ Aristotle University of Thessaloniki, Thessaloniki 54124, Greece
}

\begin{abstract}
We examine the effects of spatial inhomogeneities on irrotational anisotropic cosmologies by looking at the average properties of anisotropic pressure-free models. Adopting the Buchert scheme, we recast the averaged scalar equations in Bianchi-type form and close the standard system by introducing a propagation formula for the average shear magnitude. We then investigate the evolution of anisotropic average vacuum models and those filled with pressureless matter. In the latter case we show that the backreaction effects can modify the familiar Kasner-like singularity and potentially remove Mixmaster-type oscillations. The presence of nonzero average shear in our equations also allows us to examine the constraints that a phase of backreaction-driven accelerated expansion might put on the anisotropy of the averaged domain. We close by assessing the status of these and other attempts to define and calculate 'average' spacetime behaviour in general relativity.
\end{abstract}

PACS Numbers: 04.20.-q, 04.40.-b, 98.80.-k, 98.80.Jk

\section{Introduction}

The question of whether an inhomogeneous model of the universe behaves on the average like a homogeneous solution of Einstein's equations is a long-standing problem, directly related to the question of how to average general-relativistic spacetimes [1]-[3]. The problem lies in the generic non-linearity of general relativity and in the difficulty of establishing a unique and unambiguous way of averaging the spacetime metric without throwing away crucial information in the averaging process. A number of cosmologists have argued that the averaging problem may be central to our understanding of the recent expansion history of the visible universe. When it was suggested, recently, that structure formation could be responsible for the observed acceleration of the universe [4]-[7], averaging techniques were employed to study the dark energy problem in terms of kinematic backreaction effects from spatial inhomogeneities [8,9]. Although the idea of backreaction has been criticized, primarily on the basis of current observations [10]-[13], it is attractive in principle because it can solve the dark energy and the coincidence problems without

*email: j.d.barrow@damtp.cam.ac.uk

†email: tsagas@astro.auth.gr 
appealing to a cosmological constant, speculative quintessence field, or non-linear corrections in Einstein's theory of gravity.

General relativity has been applied to a range of averaging scales depending on the nature of the physical system under study. Cosmology applies to the largest astronomical scales, which typically extend over a significant fraction of the Hubble radius. Nevertheless, the major issue of defining a suitable averaging process remains open. When employing averaging techniques to study the backreaction of cosmological perturbations, one usually averages scalars like the density or the volume expansion rate. These become the scale-dependent parameters that are compared with observations. Averaging such scalars over a Riemannian spacelike region gives the effective dynamical sources that an observer would expect to measure. Thus, the averaged equations isolate an explicit source term that is commonly referred to as the 'backreaction' term. The latter quantifies the deviation of the average model from a given 'fitting' model, which is usually the Friedmann-Robertson-Walker (FRW) universe, though not always [14].

Here, we do not use a Friedmannian universe, but an anisotropic Bianchi-type cosmology that contains the FRW models as subcases. We consider vacuum, or dust-dominated, irrotational Bianchi models with zero or isotropic local spatial curvature, aiming to describe the effective evolution of their averaged counterparts. We do this by writing the standard average formulae in Bianchi form (i.e. with explicit shear terms in the left-hand side) and by closing the system with a propagation equation for the mean shear magnitude. Within this Bianchi-type interpretation of the averaged equations the backreaction term is expressed as a difference between the meansquare fluctuations in the expansion and shear scalars. For a vacuum background with Euclidean 3 -spaces, we find that the source term vanishes and the average equations retain their local form. When pressureless matter is introduced, however, the backreaction effects can dominate the early evolution of averaged Bianchi $I$ models and prevent the familiar Kasner-type singularity from forming. Assuming axial symmetry, we examine the 'bounce conditions' that avoid the initial singularity for different types and degrees of anisotropy. We also consider the accelerated expansion of these models and how this might constrain their average shear anisotropy.

\section{The averaging rule}

The literature contains more than one averaging method [16]-[22] that has been applied to cosmology (see [23] and more recently [8, 9] and [24]-[27]). Here, we will follow the Buchert approach which builds on the Newtonian theory, where spatial averaging is relatively simple, before extending to general relativity by confining to scalar variables [20]-[21]. Consider a general spacetime filled with irrotational pressureless matter moving along geodesic worldlines with 4-velocity $u_{a}$ (i.e. $u_{a} u^{a}=-1$ and $\dot{u}_{a}=u^{b} \nabla_{b} u_{a}=0$ ). Given a foliation of flow-orthogonal hypersurfaces, the metric of the 3-dimensional space is $h_{a b}=g_{a b}+u_{a} u_{b}$. Then, the spatial averaging of an arbitrary scalar field $\phi=\phi\left(x^{a}\right)$ over a simply-connected domain $\mathcal{D}$ is a covariant operation defined by the averaging operation $\langle\phi\rangle_{\mathcal{D}}=\int_{\mathcal{D}} \phi J \mathrm{~d}^{3} x^{\alpha} / V_{\mathcal{D}}$, where the angled brackets indicate

spatial averages, $V_{\mathcal{D}}$ is the volume of the domain in question, and $J=\sqrt{\operatorname{det}\left(h_{a b}\right)}[20]$. We also note that Greek indices take the values $1,2,3$ and Latin ones run from 0 to 3. Following [8], it helps to define an effective expansion scale factor by $a_{\mathcal{D}}=\left(V_{\mathcal{D}} / V_{\mathcal{D}_{i}}\right)^{1 / 3}$, with $V_{\mathcal{D}_{i}}$ representing the volume of the initial domain. Since the volume $V_{\mathcal{D}}$ is generally time dependent, the average 
of the time derivative of a locally-defined scalar differs from the time derivative of the average. This non-commutativity between spatial averaging and temporal evolution can be formulated in a simple mathematical rule. For a rest-mass preserving domain, the commutation between the volume-averaging and the time-evolution operators leads to [20, 21]

$$
\langle\phi\rangle_{\mathcal{D}}-\langle\dot{\phi}\rangle_{\mathcal{D}}=\langle\Theta \phi\rangle_{\mathcal{D}}-\langle\Theta\rangle_{\mathcal{D}}\langle\phi\rangle_{\mathcal{D}}
$$

where $\Theta$ is the local volume expansion rate between neighbouring worldlines. This scalar coincides with the trace of the local expansion tensor and also with that of the extrinsic curvature tensor. The right-hand side of the above rule generally introduces source terms to the averaged equations which can be interpreted as backreaction effects due to the averaging process. Such kinematic backreaction has recently been considered as a possible solution to the dark energy problem. We note that the appearance and the nature of the aforementioned source terms depends on the form of the averaged equations. Generally, the less information these equations contain the simpler (if any) the source terms are.

\section{Averaged anisotropic cosmologies}

\subsection{The local evolution}

Consider an irrotational, anisotropic, spacetime and introduce a family of (fundamental) observers living along worldlines tangent to the future-directed timelike eigenvectors of the Ricci tensor. This achieves a unique local threading of the spacetime into time and 3-dimensional space (e.g. see [28]). For a pressure-free medium, the local shear tensor propagates as [29, 30]

$$
\dot{\sigma}_{a b}=-\Theta \sigma_{a b}-\mathcal{R}_{\langle a b\rangle},
$$

where $\mathcal{R}_{\langle a b\rangle}$ is the traceless part of the locally-defined 3-Ricci tensor. The latter describes the geometry of the spatial hypersurfaces orthogonal to $u_{a}$ and satisfies the Gauss-Codacci equation. Hereafter, we will only consider spacetimes that obey the constraint $\mathcal{R}_{a b} \sigma^{a b}=0$. For example, this condition holds when the curvature of the spatial sections vanishes, or when it is isotropic. Then, the local expansion is monitored by the following three scalar propagation equations

$$
\dot{\rho}+\Theta \rho=0, \quad \dot{\sigma}+\Theta \sigma=0 \quad \text { and } \quad \dot{\Theta}+\frac{1}{3} \Theta^{2}+\frac{1}{2} \rho+2 \sigma^{2}-\Lambda=0,
$$

supplemented by the constraint

$$
\frac{1}{2} \mathcal{R}-\rho+\frac{1}{3} \Theta^{2}-\sigma^{2}-\Lambda=0 .
$$

Here, $\rho$ is the matter density, $\Theta$ is the expansion scalar, $\sigma$ is the shear magnitude, $\mathcal{R}$ is the Ricci scalar of the spatial sections, and $\Lambda$ is the cosmological constant.

We remind the reader that the adopted averaging scheme requires zero rotation to guarantee the existence of integrable spatial hypersurfaces. Also, the method has been applied to study the backreaction effects in pressure-free cosmological environments. Here, in addition to zero rotation and pressure, we assume that $\mathcal{R}_{a b} \sigma^{a b}=0$. For all practical purposes the latter is a very mild constraint and still covers all (non-rotating and pressure-free) spacetimes with flat spatial 
sections or isotropic 3-curvature. These include those Bianchi models that contain the FRW universes as subcases. Primarily, condition $\mathcal{R}_{a b} \sigma^{a b}=0$ allows us to reduce Einstein's equations to a closed set of four scalar formulae. Previous work on the backreaction effects of spatial averaging did not need a shear propagation equation and the associated sets of scalar equations were not closed (in reminiscent of the Newtonian 'gravitational paradox' - see [31]).

\subsection{The average evolution}

Because spatial averaging and time evolution do not commute, the averaged equations contain extra terms, compared to their local counterparts. By giving to the average formulae the form of a chosen set of local equations, one can interpret these extra terms as the backreaction effect of the averaging process. Here, we will write the averaged formulae in Bianchi-type form by allowing for explicit shear terms in their left-hand side. Thus, applying law (1) to the matter density, the shear magnitude, and the volume expansion, we may use Eqs. (3a)-(3c) to obtain

$$
\langle\rho\rangle^{\cdot}+\langle\Theta\rangle\langle\rho\rangle=0, \quad\langle\sigma\rangle^{\cdot}+\langle\Theta\rangle\langle\sigma\rangle=0
$$

and

$$
\langle\Theta\rangle^{\cdot}+\frac{1}{3}\langle\Theta\rangle^{2}+\frac{1}{2}\langle\rho\rangle+2\langle\sigma\rangle^{2}-\Lambda=\frac{2}{3}\left\langle(\Theta-\langle\Theta\rangle)^{2}\right\rangle-2\left\langle(\sigma-\langle\sigma\rangle)^{2}\right\rangle,
$$

respectively. Also, the volume-averaged counterpart of (4) reads

$$
\frac{1}{2}\langle\mathcal{R}\rangle-\langle\rho\rangle+\frac{1}{3}\langle\Theta\rangle^{2}-\langle\sigma\rangle^{2}-\Lambda=-\frac{1}{3}\left\langle(\Theta-\langle\Theta\rangle)^{2}\right\rangle+\left\langle(\sigma-\langle\sigma\rangle)^{2}\right\rangle,
$$

where the right-hand sides of (6) and (7) involve the identity $\left\langle(\phi-\langle\phi\rangle)^{2}\right\rangle=\left\langle\phi^{2}\right\rangle-\langle\phi\rangle^{2}$. The above set is a system of four effective Einstein equations for spatially averaged scalar variables in inhomogeneous irrotational universes with zero fluid pressure and $\mathcal{R}_{a b} \sigma^{a b}=0$. Within this environment Eqs. (5)-(7) are exact and there is no need to assume that the inhomogeneity and the anisotropy are small perturbations $[8,9]$.

Comparing Eqs. (5) to (3a) and (3b) we notice that, despite the non-commutativity between volume averaging and time evolution, the averaged formulae have preserved the form of their local counterparts. This means that

$$
\langle\rho\rangle \propto a_{\mathcal{D}}^{-3} \quad \text { and } \quad\langle\sigma\rangle \propto a_{\mathcal{D}}^{-3},
$$

where $a_{\mathcal{D}}$ is the average scale factor smoothed out over the domain $\mathcal{D}$ (with $\dot{a}_{\mathcal{D}} / a_{\mathcal{D}}=\langle\Theta\rangle / 3$ ). However, the averaging process has led to extra terms in the right-hand sides of (6) and (7), collectively given by the domain-dependent scalar

$$
\mathcal{Q} \equiv \frac{2}{3}\left\langle(\Theta-\langle\Theta\rangle)^{2}\right\rangle-2\left\langle(\sigma-\langle\sigma\rangle)^{2}\right\rangle=-\frac{2}{3}\left(\langle\Theta\rangle^{2}-\left\langle\Theta^{2}\right\rangle\right)+2\left(\langle\sigma\rangle^{2}-\left\langle\sigma^{2}\right\rangle\right) .
$$

We interpret the above as the backreaction effect of spatial averaging on pressure-free Bianchi I models, since in its absence the averaged formulae recover the form of their local counterparts (compare to Eqs. (3), (4)). Note that $\mathcal{Q}$ can be either positive or negative, and its value is decided by the amount of kinematic anisotropy. In particular, $\mathcal{Q}$ is positive when the shear mean-square fluctuation is less than a third of that in the volume expansion. 


\subsection{The kinematic backreaction term}

The form of the $\mathcal{Q}$-term depends on the specifics of the adopted cosmology. For instance, in empty models with zero cosmological constant and 3-curvature, the local shear and the expansion are related by $\Theta / \sqrt{3}=\sigma$ (see Eq. (4) earlier). This immediately ensures that $\langle\Theta\rangle / \sqrt{3}=\langle\sigma\rangle$, and subsequently that $\langle\Theta\rangle^{2} / 3=\langle\sigma\rangle^{2}$. Hence, following (9), the backreaction term vanishes identically, and Eqs. (6), (7) maintain the form of their local counterparts.

When there is matter or nonzero 3-curvature, the backreaction effects do not generally vanish. In those cases we can monitor the dynamical evolution of $\mathcal{Q}$ via a consistency/integrability condition. Taking the time derivative of (7) and then using (5), (6) we obtain

$$
\dot{\mathcal{Q}}+2\langle\Theta\rangle \mathcal{Q}=-\langle\mathcal{R}\rangle \cdot-\frac{2}{3}\langle\Theta\rangle\langle\mathcal{R}\rangle
$$

It follows that in the absence of pressure and rotation, and also for $\mathcal{R}_{a b} \sigma^{a b}=0$ in our case, the evolution of the backreaction term depends on that of the average 3 -Ricci scalar. When $\mathcal{R} \equiv 0$ or $\langle\mathcal{R}\rangle \propto\langle a\rangle^{-2}$, for example, we find that

$$
\mathcal{Q} \propto a_{\mathcal{D}}^{-6}
$$

Overall, a positive $\mathcal{Q}$ mimics the dynamics of a dark energy component and tends to accelerate the expansion, whereas a negative one acts as an effective dark matter source (see Eq. (6)). In particular, a negative $\mathcal{Q}$ behaves like an effective fluid with a 'stiff' equation of state (e.g. a free scalar field) or like an additional shear source.

\section{Backreaction effects}

\subsection{Backreaction and Kasner-type singularities}

The scalar $\mathcal{Q}$ carries the collective backreaction effects from spatial inhomogeneities. This could mean a considerable loss of information, relative to that contained in the local equations. For instance, the sign of the backreaction term is crucial but unknown. There is an advantage, however, because the compactness of $\mathcal{Q}$ makes it an easier to handle quantity. Thus, a positive $\mathcal{Q}$ was recently suggested as potential cause of late-time acceleration in FRW-type average cosmologies. Here we will consider average Bianchi-type models and examine whether analogous backreaction effects can modify (or even remove altogether) standard features of their conventional counterparts.

Integrating (3a) and (3b), we find that $\rho \propto a^{-3}$ and $\sigma^{2} \propto a^{-6}$, with the latter holding for $\mathcal{R}_{a b} \sigma^{a b}=0$. This means that shear anisotropies dominate the early evolution of these models, which approach the Kasner-type singularities of the vacuum Bianchi $I$ spacetimes (with $\Lambda=0$ ). This singular behaviour is not guaranteed for the averaged counterparts of these cosmologies because of the backreaction term and particularly because of (11). Given that both $\langle\sigma\rangle^{2}$ and $\mathcal{Q}$ propagate as $a_{D}^{-6}$ while $\langle\rho\rangle \propto a_{D}^{-3}$, the early stages of the averaged kinematics is dominated by the sum $\mathcal{Q}-2\langle\sigma\rangle^{2}=2\left\langle(\Theta-\langle\Theta\rangle)^{2}\right\rangle / 3-2\left\langle\sigma^{2}\right\rangle$. When the latter is negative and $\langle\Theta\rangle<0$ initially, the collapse will proceed unimpeded and $\langle\Theta\rangle \rightarrow-\infty$ within a finite amount of time. Otherwise the averaged model should bounce before reaching the singularity. 
Additional information is obtained by expressing $\mathcal{Q}$ in terms of the averaged individual expansion rates. Assume, for example and also for the sake of simplicity, that the inhomogeneous spacetime is axially symmetric and has zero 3-curvature. Then $\Theta=2 \alpha+\beta, \rho=\alpha(\alpha+2 \beta)$ and $\sigma=(\alpha-\beta) / \sqrt{3}$. In this notation $\alpha=\dot{a} / a$ and $\beta=\dot{b} / b$ are the expansion rates along the two main axes, with $a$ and $b$ representing the associated scale factors of the axisymmetric Bianchi $I$ universe $[32,33]$. Within this environment, expression (9) reads

$$
\mathcal{Q}=2\left(\left\langle\alpha^{2}\right\rangle-\langle\alpha\rangle^{2}\right)+4(\langle\alpha \beta\rangle-\langle\alpha\rangle\langle\beta\rangle)
$$

allowing for $\mathcal{Q}$ to be positive, negative, or zero. ${ }^{1}$ Hence, if the expansion is nearly isotropic (i.e. for $\alpha \simeq \beta$ ), the bounce condition $\mathcal{Q}>2\langle\sigma\rangle^{2}$ is satisfied when $\left\langle(\alpha-\langle\alpha\rangle)^{2}\right\rangle>0$. For highly anisotropic expansion, with $\alpha \gg \beta$, we have $\langle\sigma\rangle^{2} \simeq\langle a\rangle^{2} / 3$. In this case, we will have a bounce when the mean-square fluctuation of $\alpha$ is greater than $\langle\alpha\rangle^{2} / 3$. Finally, for $\alpha \ll \beta$, the backreaction effects can prevent the $\langle\Theta\rangle \rightarrow-\infty$ singularity provided that $\langle\alpha \beta\rangle-\langle\alpha\rangle\langle\beta\rangle>\langle\beta\rangle^{2} / 6$.

It is well known that the Mixmaster behaviour of the Bianchi $I X$ models consists of a sequence of chaotically alternating Kasner-like time intervals where the collapsing and expanding scale factors interchange (e.g. see [32] and also [34, 35]). The possibility that backreaction effects due to spatial averaging could alleviate the Kasner-type singularities of the Bianchi $I$ models, raises the question as to whether the same effects could also modify the Mixmaster regime of the Bianchi $I X$ spacetimes. The possible role of stochastic spatial fluctuations was discussed in [36]. It was argued there that the cumulative stochastic effects from nearby gravity-wave perturbations could transform the local Einstein equations into stochastic differential equations with significant damping effects on the chaotic oscillations. Here, we see the cumulative effect of the stochastic inhomogeneities mimics a scalar field of the sort that is known to stop the continuation of Mixmaster oscillations on approach to the initial singularity (if it exists) [37]. Based on these we conjecture that, within the framework of the Buchert averaging scheme, Mixmaster oscillations will be unstable at early times.

\subsection{Accelerated expansion and average anisotropy}

Let us suppose that the aforementioned backreaction effects lead to the acceleration of the averaged domain. The presence of nonzero mean shear in the equations allows us to consider the constraints imposed on the average anisotropy of the region. Using the scale-dependent average scale factor $a_{\mathcal{D}}$, defined so that $\dot{a}_{\mathcal{D}} / a_{\mathcal{D}}=\langle\Theta\rangle / 3$, the averaged Raychaudhuri equation (see (6)) takes the form

$$
3 \frac{\ddot{a}_{D}}{a_{D}}=-\frac{1}{2}\langle\rho\rangle-2\langle\sigma\rangle^{2}+\Lambda+\mathcal{Q} .
$$

Here, the backreaction term can be interpreted as an effective energy-density term or an effective shear term. ${ }^{2}$ In any case, and in the absence of a cosmological constant, the volume expansion

\footnotetext{
${ }^{1}$ When $\alpha=-2 \beta$ expression (12) ensures that $\mathcal{Q}=0$ and therefore the absence of any averaging backreaction effects. This is expected since $\alpha=-2 \beta$ means $\rho=0$ and we know that $\mathcal{Q}=0$ in the vacuum models (see $\S 3.3$ ).

${ }^{2}$ One can introduce a chosen 'background' to the average equations by defining a suitable reference model. Thus, the time-dependent expansion and shear fields, $H=H(t)$ and $\Sigma_{a b}=\Sigma_{a b}(t)$ respectively, define a homogeneous background into the averaged formulae [20]. Inhomogeneous deviations from these reference fields are considered by setting $\Theta=H+\hat{\Theta}$ and $\sigma_{a b}=\Sigma_{a b}+\hat{\sigma}_{a b}$. This type of decomposition is not essential for our
} 
of the averaged domain will accelerate provided that

$$
\mathcal{Q}>\frac{1}{2}\langle\rho\rangle+2\langle\sigma\rangle^{2}
$$

which here holds for zero or isotropic 3-curvature (strictly speaking as long as $\mathcal{R}_{a b} \sigma^{a b}=0$ ). We note that if $\mathcal{Q}$ is negative, namely if the mean square fluctuation in the shear is greater than a third of the mean square fluctuation in the volume expansion (see (9)), the domain will decelerate instead of accelerating. We have no information on the sign of the backreaction term however. Because of this ambiguity, early on, kinematic backreaction was also suggested as an effective dark-matter source [38].

Putting these matters aside, we will use our Bianchi-based interpretation of the backreaction effects and the form of the average equations to constraint the mean anisotropy of the accelerating region. To proceed we introduce the following domain-dependent, dimensionless set:

$$
\Omega_{\rho}=\frac{3\langle\rho\rangle}{\langle\Theta\rangle^{2}}, \quad \Omega_{\sigma}=\frac{3\langle\sigma\rangle^{2}}{\langle\Theta\rangle^{2}}, \quad \Omega_{\mathcal{R}}=-\frac{3\langle\mathcal{R}\rangle}{2\langle\Theta\rangle^{2}}, \quad \Omega_{\mathcal{Q}}=-\frac{3 \mathcal{Q}}{2\langle\Theta\rangle^{2}}
$$

and

$$
\Omega_{\Lambda}=\frac{3 \Lambda}{\langle\Theta\rangle^{2}} .
$$

We note that $\Omega_{\rho}, \Omega_{\sigma}$ and $\Omega_{\Lambda}$ (assuming $\Lambda>0$ ) are positive definite, while $\Omega_{\mathcal{R}}$ and $\Omega_{\mathcal{Q}}$ can take negative values. In particular, $\Omega_{\mathcal{Q}}$ is negative when $\mathcal{Q}$ is positive, which happens when the mean-square fluctuation of the shear is small compared to that of the expansion (see (16d)). In terms of these parameters, and after setting $\Lambda=0$, condition ( 7 ) reads

$$
\Omega_{\rho}+\Omega_{\sigma}+\Omega_{\mathcal{R}}+\Omega_{\mathcal{Q}}=1
$$

while (15) becomes

$$
\Omega_{\mathcal{Q}}<-\frac{1}{4} \Omega_{\rho}-\Omega_{\sigma}
$$

Combining this result with constraint (18) we can recast the condition for accelerated expansion, without any $\Omega_{\sigma}$ and $\Omega_{\mathcal{Q}}$ contributions, into the following more familiar form $[8,9]$

$$
\Omega_{\rho}+\frac{4}{3} \Omega_{\mathcal{R}}>\frac{4}{3} .
$$

We may constrain the shear anisotropy of the accelerating averaged domain by combining conditions (18) and (20). For instance, setting $\Omega_{\mathcal{R}}=0$ and substituting (20) into (18) gives

$$
0<\Omega_{\sigma}<-\frac{1}{3}-\Omega_{\mathcal{Q}} .
$$

This is a self-consistent condition as long as $\Omega_{\mathcal{Q}}<-1 / 3$, or equivalently $\mathcal{Q}>2\langle\Theta\rangle^{2} / 9$ (see definition (16d)). Following (9), the latter requires the mean-square fluctuations in the volume expansion to exceed those in the shear by at least $2\langle\Theta\rangle^{2} / 9$. Note that, when $\Omega_{\mathcal{Q}} \rightarrow-1 / 3^{-}$, accelerated expansion occurs when there is minimal shear anisotropy (i.e. for $\Omega_{\sigma} \rightarrow 0$ ).

purposes. If adopted, however, the averaged Raychaudhuri equation (e.g. see (13)) will take the form

$$
3 \frac{\ddot{a}_{D}}{a_{D}}=-\frac{1}{2}\langle\rho\rangle+\frac{2}{3}\left(\left\langle\hat{\Theta}^{2}\right\rangle-\langle\hat{\Theta}\rangle^{2}\right)-2 \Sigma^{2}-2\left\langle\Sigma_{a b} \hat{\sigma}^{a b}\right\rangle-2\langle\hat{\sigma}\rangle^{2}+\Lambda,
$$

with an analogous expansion for (7). Clearly, when the 'background' is isotropic all the $\Sigma$-terms vanish identically. 


\section{Discussion}

The averaging problem in general relativity and cosmology is an issue of major importance that remains largely unresolved. As yet and despite the efforts there is no general consensus on what the approach to spatial averaging should be. Generally, averaged inhomogeneous spacetimes are known to evolve differently from the standard cosmological model, represented by the FRW universe. It is also known that spatial averaging leads to effective source terms in the Einstein equations, analogous to Reynolds stress terms in turbulent fluid flows, which carry what are commonly referred to as backreaction effects. In fact, these effective dynamical sources are what an observer would expect to measure. However, although the measurements take place in the real inhomogeneous world, their interpretation depends on the chosen fitting cosmological model. So far, the latter has been the Friedmann universe and the studies have shown that spatial averaging can seriously modify its standard dynamics, particularly its expansion rate. In view of the uncertainty about the averaging procedure and its physical consequences, it is important to interpret spatial averaging within a range wider than the FRW models.

In this study we assumed a Bianchi-type cosmology with pressure-free matter and zero or isotropic 3-curvature. Our intention was to calculate the effective evolution of the averaged counterparts of these simple anisotropic models. Allowing for explicit average-shear terms in the equation, we recast the standard formulae in a closed Bianchi-type form. Thus, in our case, the backreaction source term is given by the difference between the mean-square fluctuations in the expansion and the shear scalars. For a vacuum fitting model with Euclidean 3-spaces, we found that the source term vanishes and the averaged equations retain their local form. When pressureless matter is introduced, however, the backreaction effects can dominate the early evolution of averaged Bianchi $I$ models and prevent the familiar Kasner-type singularity from forming. Assuming axial symmetry, we have considered the 'bounce conditions' for different types and degrees of anisotropy. The possibility of avoiding the Kasner regime in averaged inhomogeneous Bianchi $I$ universes, raises the question as to whether the same backreaction effects could also alleviate the Mixmaster behaviour in averaged Bianchi $I X$ spacetimes. By giving a Bianchi-type form to the average equations, we were allowed to consider the limits that a period of backreaction triggered accelerated expansion might put on the anisotropy of the averaged domain. Our results show that these constraints are largely compatible with the observed high isotropy of the universe.

When averaging relativistic spacetimes one should always keep in mind that there is no unique approach so far (e.g. see [24]-[26]). In addition to that, there is another central concern that besets all averaging methods and creates uncertainty about their conclusions (e.g. see [13]). Averaging takes an ensemble of spacetimes $\left(M_{i}, g_{i}\right)$ - all of which satisfy the standard Einstein equations - and introduces a procedure by which we can define an 'average' spacetime $(\bar{M}, \bar{g})$ associated with them. This process necessarily loses information about the $\left(M_{i}, g_{i}\right)$ which could lead to spurious astrophysical conclusions about the behaviour of $(\bar{M}, \bar{g})$. For instance, if every member of the ensemble $\left(M_{i}, g_{i}\right)$ is a dust-filled FRW universe with an initial singularity, then it seems very peculiar if an 'average' of these behaviours, every one of which is singular, could give rise to a non-singular 'average' universe. Yet, this is in effect what is happening in many of the studies of the averaged behaviour of FRW universes. Each member of the ensemble obeys the strong-energy condition and possesses decelerating expansion, but the averaged universe 
accelerates and in a sense it effectively violates the strong energy condition. This is exactly the situation that is being appealed to in a number of recent studies, and also emerges in our examples. It remains to be seen whether the extension of the averaging procedure to deal with non-scalar quantities can produce a consistent resolution of this information-loss problem by controlling the type and the quantity of the lost information. We know that the process of perturbing or averaging the Einstein equations is not a sequence of operations that commutes. The definition of an averaged spacetime would be the result of solving the Einstein equations in general and then averaging the solution. In practice, we average them and then solve the averaged equations. This suggests that we might narrow the gap between the two results of these approaches by averaging an exact inhomogeneous cosmological solution of Einstein's equations and comparing the result with the averaging process applied to a FRW ensemble. Our analysis has been made in this same spirit of testing the self-consistency of an averaging procedure in new situations to determine whether we can rely upon the more familiar ones.

\section{Acknowledgments}

We would like to thank Thomas Buchert, Julien Larena and Aseem Paranjape for their comments. CGT also wishes to thank the Centre for Mathematical Sciences at Cambridge University, where part of this work was conducted, for their hospitality.

\section{References}

[1] Shirokov M F and Fisher I Z 1963 Sov. Astron. J. 6699

[2] Ellis G F R 1984 In General Relativity and Gravitation ed B Bertotti, F de Felice and A Pascolini (Dordrecht: Reidel) p 215

[3] Ellis G F R and Stoeger W 1987 Class. Quantum Grav. 41697

[4] Räsänen S 2004 JCAP 0402003

[5] Kolb E W, Mataresse S, Notari A and Riotto A 2005 Phys. Rev. D 71023524

[6] Barausse E, Mataresse S and Riotto A 2005 Phys. Rev. D 71063537

[7] Kolb E W, Mataresse S, Notari A and Riotto A 2005 Preprint hep-th/0503117

[8] Buchert T 2006 Class. Quantum Grav. 23817

[9] Räsänen S 2006 Class. Quantum Grav. 231823

[10] Geshnizjani G, Chung D J H and Afshordi N 2005 Phys. Rev. D 72023517

[11] Buchert T 2005 Class. Quantum Grav. 22 L113

[12] Giovannini M 2006 Phys. Lett. B 6341

[13] Ishibashi A and Wald R M 2006 Class. Quantum Grav., 23235 
[14] Paranjape A and Singh T P 2006 Class. Quantum Grav. 231

[15] Ellis G F R and Buchert T 2005 Phys. Lett. A 34738

[16] Isaacson R A 1968 Phys. Rev 1661263

[17] Bildhauer R M and Futamase T 1991 Gen. Rel. Grav. 231251

[18] Zalaletdinov R M 1992 Gen. Rel. Grav. 241015

[19] Zalaletdinov R M 1997 Bull. Astron. Soc. India 25401

[20] Buchert T and Ehlers J 1997 Astron. Astrophys. 3201

[21] Buchert T 2000 Gen. Rel. Grav. 32105

[22] Buchert T 2001 Gen. Rel. Grav. 331381

[23] Futamase T 1996 Phys. Rev. D 53681

[24] Coley A A, Pelavas N and Zalaletdinov R M 2005 Phys. Rev. Lett. 95151102

[25] Coley A A and Pelavas N 2006 Phys. Rev. D 74087301

[26] Coley A A and Pelavas N 2006 Preprint gr-qc/0607079

[27] Buchert T, Larena J and Alimi J-M 2006 Class. Quantum Grav. 236379

[28] Ellis G F R and van Elst H 1999 in Theoretical and Observational Cosmology ed M LachiézeRay (Dordrecht: Kluwer) p 1

[29] Tsagas C G and Maartens R 2000 Class. Quantum Grav. 17, 2215

[30] Barrow J D and Tsagas C G 2005 Class. Quantum Grav. 221563

[31] Barrow J D and Götz 1989 Class. Quantum Grav. 61253

[32] Landau L and Lifshitz E M 1975 The Classical Theory of Fields (Oxford: Pergamon)

[33] Barrow J D 1997 Phys. Rev. D 557451

[34] Barrow J D. 1982 Phys. Rep. 851

[35] Chernoff D and Barrow J D 1983 Phys. Rev. Lett. 50134

[36] Barrow J D 1984 in Classical General Relativity eds W B Bonner, J N Islam and M A H MacCallum (Cambridge: Cambridge University Press) p 25

[37] Belinskii V and Khalatnikov I 1973 Sov Phys JETP 36591

[38] Buchert T. 1996 in Mapping, Measuring and Modelling the Universe ed P Coles (San Francisco: Astronomic Society of the Pacific) 94349 